\title{
PAKISTAN HEALTHCARE INFRASTRUCTURE AND COVID-19: A CASE FOR ISOLATION AND QUARANTINE FACILITIES OPTIMIZATION IN THE CONTEXT OF PAKISTAN
}

\author{
Omer Shujat Bhatti* \\ Asad Ghufran**
}

\begin{abstract}
COVID-19 is one of the largest pandemic that hit the world at the end of 2019 and took the globe by a storm. With almost seven hundred fifty thousand affected and above thirty five thousand deaths at the time of writing this paper, it was declared by World Health Origination (WHO) a major global health crisis. Pakistan, a developing South Asian country having sixth largest population in the world having fragile and poor healthcare infrastructure also got hit badly with above one thousand six hundred affected and more than twenty deaths till the time of writing. With such a large health burden at stake and closing of all major socio-economic activities, with having very limited quarantine and isolation facilities, there was a dire need to explore the gap of existing healthcare infrastructure to cope with current circumstances.
\end{abstract}

In order to explore the current context with reference to the COVID-19 health burden, existing infrastructure and facilities were explored through literature review and documented against the criteria and outlined by WHO, with respect to quarantine and isolation facilities. Development of a basic concept about nature of COVID-19 was explored with respect to Pakistan and its spread in the country. Existing actions taken by the governing concerned bodies and future planning were also explored. It was concluded through comparative analysis and exploration of the current line of actions by the governing bodies, that existing infrastructure fails to fulfill the need of the current pandemic situation and transformation of existing healthcare facilities and other allied buildings, like hostels and educational institutions, would not help in development of quarantine and isolation facilities. Infact they will create higher level risks for contamination and management of the disaster at hand, since these do not fulfill the set criterion for isolation and quarantine facilities with functional and spatial aspects. A two stream approach is proposed to handle the current situation with focus on using existing facilities at one hand with limited usage for quarantine and isolation, while developing new functional facilities using fast construction systems of pre-fabricated construction this will enable quick delivery of such infrastructure to manage the COVID-19 in Pakistan with context and climatic sensitive designs. Proposed design interventions associated with quarantine and isolation units were further explored and using standardized cargo containers design solutions were proposed.

Keywords: COVID-19, Pakistan, Isolation Wards, Quarantine, Healthcare Design, Infection Control and Prevention.

\section{INTRODUCTION}

Large scale epidemics and spread of diseases across nations and countries have always caused threat to humanity. Since the history of mankind, such epidemics have caused great danger and loss to the society through destroying human capital and large scale devastation of socio-economic infrastructure (Bedford et al., 2019). Twenty first century is no exception, with SARS (Severe Acute Respiratory Syndrome) in 2002, Swine Flu (Pandemic H1N1/09) in 2009, Ebola in 2013 and COVID-19 (Novel Corona virus Pandemic) in 2019 (CDC, 2020a). It is evident that these events are large enough to jolt down the existing healthcare and socio-economic basis of the society to converge all efforts towards their eradication when saving human lives becomes the most significant objective of the time (Columbus, Brust and Arroliga, 2020). COVID-19 was first

\footnotetext{
* Omer Shujat Bhatti, Research Associate, Department of Enviornmental Design, Health \& Nutritional Sciences, AIOU, Islamabad, Pakistan. Email correspondance: omer.shujat@alou.edu.pk

** Dr. Asad Ghufran, Assistant Professor, Department of Environmental Science IIUI, Islamabad, Pakistan.
} 
discovered in December 2019 Wuhan, China and later spread to more than one hundred sixty countries of the world resulting in the death toll to rise to above eleven thousand by mid March 2020 (Khan, el. al., 2020). Thus facilities to be able to adapt to these demanding times where facilitation, treatment, isolation, quarantine and control of the disease became a challenge even for the most advanced and developed countries (Brüssow, 2020). In developing countries like Pakistan, quarantine facilities were not available and no prior planning was made to control and restrict movement, the spread was mainly spontaneous. It lead to serious consequences with multiple provinces getting effected. Educational institutions were forced to shut down, academic activities were hindered, social and religious gatherings were prohibited and shopping centers were directed to close inthe early months, to avoid further spread of disease (Ahmad, el. al., 2020).

Pakistan has a fragile healthcare infrastructure with almost six beds per ten thousand population (WHO,2020). Facing COVID-19 was indeed one of the greatest challenge for the whole nation, as well as governing agencies and governments. The disease spread could be prevented through control measures on large scale, yet the spread due to lack of awareness, poor and inadequate diagnostic facilities, fragile socio-economic conditions of the majority of population, lack of healthcare facilities and infrastructure evident to happen (NIH, 2020b). Hence there was a strong need to evaluate how the existing facilities could be optimized for these current needs to address this life threatening situation all across the country.

\section{LITERATURE REVIEW}

\section{Existing Pakistan Healthcare system and current scenarios}

According to World Health Organization (2020), Pakistan consists of a diverse healthcare infrastructure comprising of two major components i.e. governmental and private hospitals. Though healthcare is the primary responsibility of the Federal and Provincial governments, yet the private share of facilities is no less. Service delivery is mainly organized and modulated through preventive, promotive, curative and rehabilitative efforts, with preventive and promotive efforts mainly through multiple organizations, including government run via different programs and projects (Bearman et al., 2020). These infrastructural facilities include basic health units, rural health units, district health units, secondary and tertiary level hospitals, and teaching hospitals, (Stahl and Falaschetti, 2012).

Details of public facilities, healthcare workforce (as of 2017) and population based statistics are outlined in Table 1.

However, it is evident from recent literature exploration that healthcare system and infrastructure fails to meet the needs of Pakistan. Some of the major key findings pointout that the government of Pakistan spends only $0.4-0.6 \%$ of its budget on health, $78 \%$ and above people spend on medical treatment out of their own pockets. Health workforce is way below set standards by WHO and absence of programs for non-communicable diseases at provincial and federal levels makes the situation worse. Regulatory arrangements for medical treatment and medication itself are unsatisfactory. There is lack of medical, diagnostics and health promotion educational programs. Maternal and infant mortality rates are very high i.e. $260 / 100,000$ deaths and 61.27 per 1,000 live births, respectively, $19 \%$ of general population and $30 \%$ of children under the age of five years are suffering from mal-nutrition. Hence it can be easily stated that Pakistan is under extreme pressure to cope up with health and medical needs and facing another global health challenge in the form of COVID-19 is a nightmare for the public in general and governing bodies and concerned departments in specific.

Table-1: Public Facilities, Healthcare Workforce (as of 201) and Population Based Statistics.

\begin{tabular}{|c|l|c|l|c|}
\hline S. No. & \multicolumn{1}{|c|}{ Facility } & Count & \multicolumn{1}{c|}{ Type } & Count \\
\hline 1 & Hospitals & 1201 & Registered Doctors & 195896 \\
\hline 2 & Basic Health Units & 5518 & Registered Dentists & 18333 \\
\hline 3 & Rural Health Centers & 683 & Registered Nurses & 99228 \\
\hline 4 & Dispensaries & 5802 & Population per Doctor & 1300 \\
\hline 5 & Maternity and Child Health Centers & 731 & Papulation per Dentist & 10658 \\
\hline 6 & TB Centers & 347 & Papulation per Bed & 1584 \\
\hline 7 & Beds in all Above Mentioned Facilities & 123394 & & \\
\hline 8 & Lady Health Workers & 95000 & & \\
\hline
\end{tabular}

Source: Khan, 2019 and Stahl and Falaschetti, 2012. 


\section{COVID-19 AND PAKISTAN}

At the outset of the government of Pakistan took initiatives in the form of allocating designated hospitals for infection patients, developing quarantine and isolation facilities through designated healthcare and setting up of temporary facilities (NIH, 2020a), infection control protocols establishments, closing of malls, educational institutions and large scale banning and restrictions of public gatherings, restricting cross-provincial movements, testing and screening of anticipated patients and travelers and developing emergency helpline numbers and center (NIH, 2020b).

\section{Concept of Quarantine and Isolation for COVID-19}

Quarantine is an old concept of keeping people away from other people who have either been in certain contact or have potentially been in contact with people suffering from transmittable pathologies (Khan et al., 2020). This criteria is followed uptil it is certain that the kept away person is not suffering from any such illness which can be part of any epidemic. The concept roots back in culture and history throughout the world where epidemics have evolved. Previously it was mainly referred to as specific time of isolation when people were kept away to ensure they are not effected from any communicable disease to restrict their movements. It is applicable to animals, humans and even things (Conti, 2016).

Isolation is mainly used for people who have been certified/identified that have been exposed to the epidemic or disease and have the potential to carry it on or pass on to other individuals. Isolation restricts the movement of sick persons to help stop the spread of certain diseases. It has previously been used in history, recently in cases of MERS and SARS spread and have provided evidence to support the containment of epidemics and communicable diseases (CDC, 2020b).

To judge the severity and the scale of impact in pandemics/epidemics, two major variables that contribute include severity and transmissibility. Centre for Diseare Control (CDC) and Prevention, USA and WHO have worked multiple plans and guidelines for the isolation of patients and quarantine to ensure detainment of the epidemic.

Proposed Quarantine measures for COVID-19 as per developed by CDC and WHO were mainly as follows (United Nations, 2020):

Countries should communicate and socialize these measures to avoid panic and ensure compliance. Countinies should provide clear, precise and updated guidelines, engage with communities and establish reliable communication channels. Countinies should provide patients with support in the form of medical, psychological, social and mental help along with food, shelter, water and treatment. Aspects of cultural, geographic and socio-economic factors should be evaluated all the time. Setting for quarantine should be appropriate for the number of people to be quarantined with minimum infection prevention and control measures and minimum requirements should be monitored for health of quarantined persons for the designated period.

For people within quarantine, spacious single room with ensuite toilets and least spacing of one meter gap between beds, adequate air ventilation, filtration systems, wastemanagement protocols, increased social distancing, provision for medication, food, water and hygiene are to be given. Protection for personal belongings, communication in language of patient preference, consultation, psycho-social support, communication setup with family for support and contact information incase they get contaminated/infected are also to e provided. Special consideration for elderly, women and children has to be made. Some of the anticipated settings could include hotels, dormitories and allied facilities. Any one developing symptoms of the disease may immediately be treated for COVID-19 prior to shifting to isolation wards or allied treatment facilities.

Necessary establishment of Infection Protection and Control (IPC) facilities (design based) and developing policies, procedures and protocols as per the contextual needs of the quarantine and local climatic conditions are also neccesary. Environmental controls play a vital role in managing quarantine. Using disinfectant clean touchable surfaces regularly including beds, tables, furniture, clothing washed at 60-90 degree Celsius is a pre requisite. Waste should be disposed off in sanitary landfill and optimum disposable items should be used. Continuous medical observation to be adopted and keen observational systems should be incorporated.

With respect to creation of isolation ward/space for the COVID-19 patients, the guidelines developed by the CDC (2020) mainly include the following aspects (CDC, 2020a): All preparation should be completed prior to patients arrival with staff and facility prepared for it. There should be limited entry points along with adherance to respiratory and allied hygiene practices for infection control. Installation of physical barriers with establishments of triage stations and isolation facilities is a pre-requisite. Separate isolation based waiting 
and examination room are to be provided. The spaces for Healthcare service providers (HCP) should be separated with inlets to isolation where deemed necessary. COVID19 patient or suspected patient to be given separate room or to be share between two patients only with attached bathroom. Air infection isolation rooms (AIIR) should be designated for patients under aerosol-generation procedures with negative pressure to relative surroundings and there should be six air changes minimum per hour. Unidirectional air flow with hepafilters are recommended. Limited transport and movement of patients is to be ensured. Medical equipment for the patients should be dedicated and not shared with other units within healthcare facility. Management of laundry, food service utensils and medical waste should also be performed in accordance with routine procedures.

Though these are some of the best practices set forth yet for a country with huge population and fragile healthcare infrastructure, following these would be nightmare since no major isolation and quarantine facilities have been established in recent times (CDC, 2020a). It is evident that due to a weak approach towards the COVID-19, the existing scenario will worsen and the overall number of fatalities and infected patients will multifold. Hence to opt for quarantine and isolation facilities, contextual base analysis is a must, to cover the spread of COVID-19 and ensure maximum safety of the public in general and engaged people in managing these facilities and patients in specific.

\section{Developing Facilities for Quarantine and Isolation of COVID-19 Patients in Pakistan}

In the midst of the existing scenarios of COVID-19 in Pakistan, the governing regimes and bodies have taken the

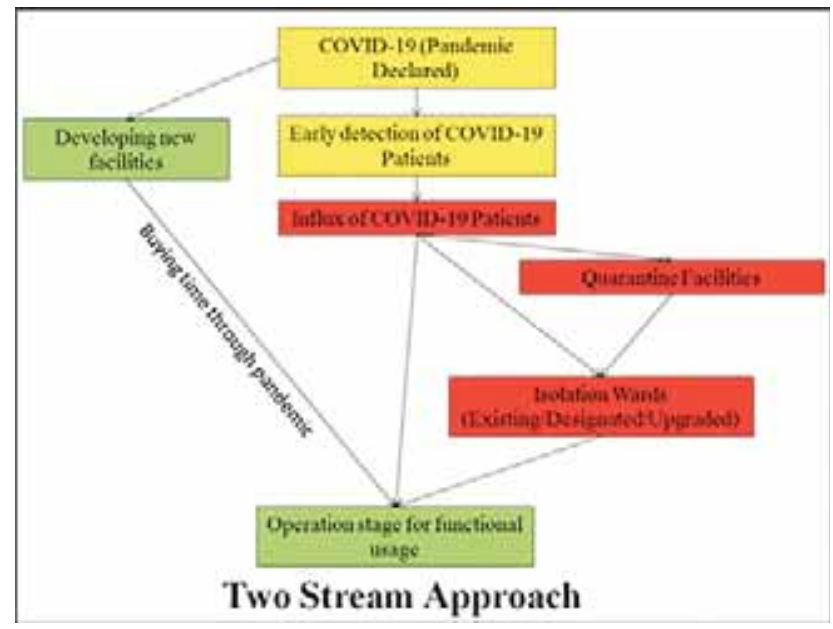

Figure-1: Two Stream Approach for Handing the Challenge of COVID19. step to develop isolation wards and quarantine facilities all across the country. Some of the hospitals have already been designated for the purpose. The patients have to follow strict measures for identification, diagnosis, treatment, release and in worse cases fatality. Measures are to be followed to take dead bodies to a graveyard without any religious or social rituals. Since existing hospitals and facilities are not developed on the same principles as that of the isolation or quarantine facilities. Designating allied spaces like large scale halls for the stated purpose would require more preparation, staff and facilities to transform these into specialized units for isolation and quarantine. Hence, there are two major options that could be proposed, based on the existing explored literature and case studies from multiple hospitals of China and other countries fighting back (Figure $1)$.

One option for setting up qurantine centers could be hotels, with each room connected with a bathroom designated for the same room with one patient in each room. Since these facilities are limited and rooms are usually considered as luxurious item for the travelers, these spaces would not be able to be used in an optimum fashion. Schools and other dormitories could be an alternate, but it has to be ensured that since patients would not be able to have toilets/washrooms each attached with a room, it would be very hard to disinfect and control infection in the common corridors. It will be more critical for the healthcare staff and services providers, to monitor the situation since they will be facing extreme risk of contamination.

Apart from hotels and schools, one option could be using the hospital units that are separated from the main hospital. These may include facilities for staff and other academic units. Since these will be within the premises of the healthcare setting, managing them would be easy as compared to the other options for which more staff would have to be acquired and transported in multiple shifts. As per the Advisory from NIH (NIH, 2020b), no major guidelines have been set for the quarantine areas.

Learning from experiences of Wuhan, China and previous fights with epidemics of MERS and SARS, it can be stated that developing designated facilities with proper design and plan can help fight the outbreack better. The Chinese opted for development of pre-fabricated structures for two hospitals in Wuhan to ensure that the burden of disease is not shared with other hospitals. Using the existing forms of prefabricated construction, like shipping containers or allied structures can be a good option for developing these facilities. 


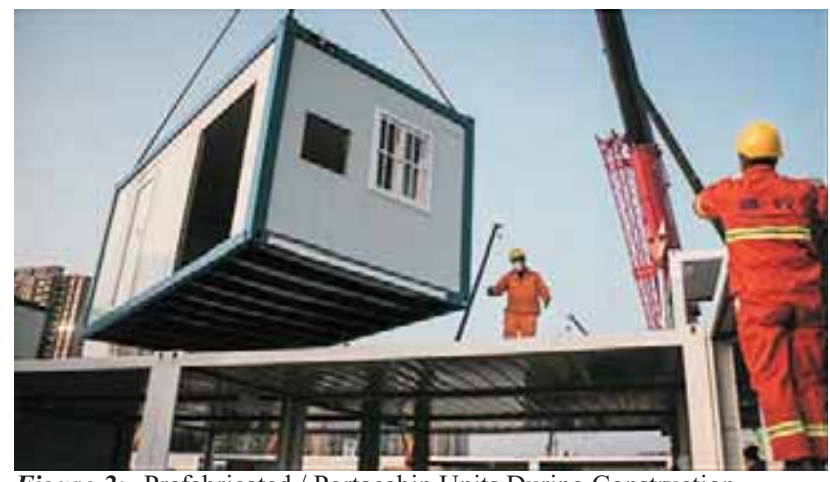

Figure-2: Prefabricated / Portacabin Units During Construction. (Source: Wuhan, 2020)

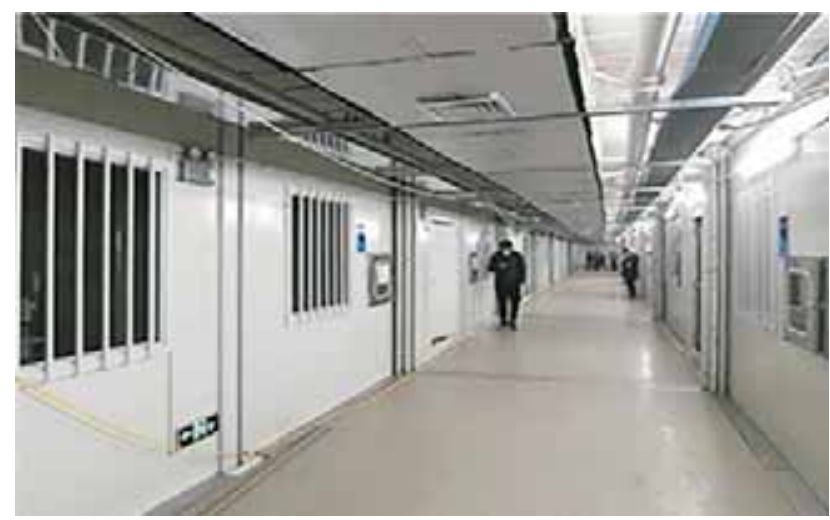

Figure-4: Separate Corridors for staff and patients in Wuhan Hospital. Source: Wuhan, 2020

There is a need to opt for two streams based approach (Figure 1) in which new facilities are targeted with focus on isolation and healthcare for COVID-19 patients. This should be initiated at multiple healthcare institutions, including major hospitals, with the help of prefabricated structures and other modes of fast construction mechanisms, while on the other hand the existing designated spaces / infrastructure can be used for isolation and social distancing. With the passage of time, new structures could be ready to take more patients, while existing facilities can be giving support to patients all along.

Following are some of the major proposed aspects to be covered and taken into consideration while opting for new facilities or upgradation/optimization/modification of existing facilities to be used as isolation wards:

1. Incorporate as many aspects of design as discussed above, as per the guidelines provided by CDC for quarantine and isolation facilities.

2. All new facilities to focus on prefab-construction systems and opt for quick fabrication of systems on site (Figure 2).

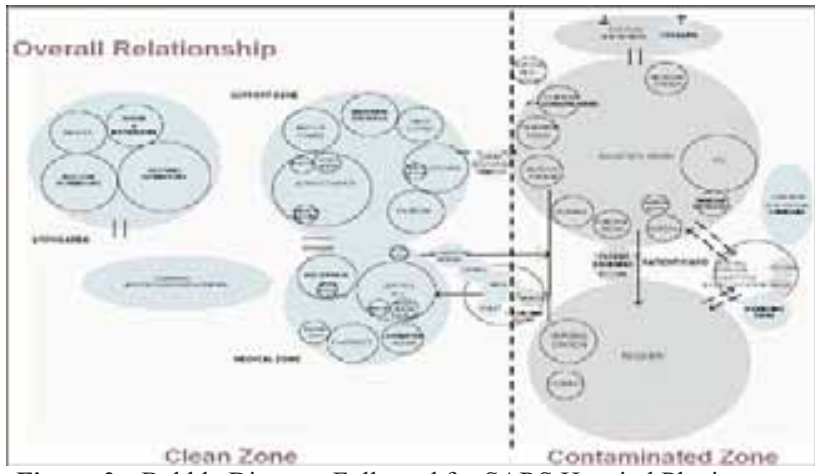

Figure-3: Bubble Diagram Followed for SARS Hospital Planing. Source: Tsou, et. al., 2004

3. Selected sites should be within the premises of an already existing healthcare facility / hospital so people can easily access and have awareness of the geographical location. It should have a new/separate gate and the premises should have clear signage for people with COVID-19 only (Figure $3)$.

4. The facility should have a separate entrance for medical staff and administration, along with medical supplies and support/allied engineering systems.

5. Designated Triage should be established to baseline the criticality of the patient. It would act as a welcome mat and first line of defense/interaction. Once the basis of the tagging is done in the triage, patient needs to be taken to the anticipated isolation facility or elsewhere. Associated facilities with the triage should be incorporated to ensure that facilitation may be completed and can lead to allied departments when required.

6. As per WHO and CDC guidelines, PPE and IPC should be maintained in all the spaces within the facility. Staff must adhere to devised rules and policies.

7. Separate corridors for the staff should be developed so they do not enter the patient designated spaces/rooms unless it deemed necessary (Figure 4).

8. Patients to be placed in negative pressure single rooms with attached toilets. Separate heating, ventilation air conditioning (HVAC) and plumbing systems should be designed to ensure waste and air flow does not come back into the internal spaces. Dedicated wards could also be used to ensure that is there some physical partition between beds with gap of atleast two meters or seven feet (Figure 5). 9. Double side opening based cabinets should be used so that caregivers do not enter the patient rooms (Figure 6). 10. It should be ensure that either single-use and disposable or dedicated equipment (e.g., stethoscopes, blood pressure cuffs, and thermometers) is present in each room. 11. Admitted patients who donot require suctioning, should be placed under droplet and contact precautions. 
12. Admitted patients who require suctioning, should be placed under airborne isolation areas with contact precautions. 13. If negative pressure isolation is not available then placed in a room with ample ventilation. A fan facing away from the door, towards the outside of the building is encouraged, if possible.

14. Patients can be moved out of isolation only when symptoms improve and two consecutive swabs (sent one day apart) are negative.

15. Laundry, food service utensils and medical waste should be managed in accordance with safety routine procedures. 16. With respect to the local context, issues of cultural and religious aspects need special attention. There should be provision for communication with families using modern technologies. Provision for prayer space and medical/personal counseling with focus on attendants and families of the patients should be provided.

17. Safety and security of the premises should be entsured and only concerned personnel may enter the premises. 18. Transportation protocol within the facility should be ensured to help the HCW perform all operations and avoid risk of contamination.

19. In case of a fatality, defined route of transfer of deceased/deadbody from facility should be nsured along with defined specific protocols for burial.

20. Most significantly, it should be ensured that infection control and prevention is managed throughout the quarantine or isolation centers.

\section{DESIGN CONSIDERATIONS}

Healthcare facilities for quarantine and isolation are highly technical in their aspects of usage and maintenance. It is based on strict levels of movement and spatial configurations, that require extensive design interventions. In Pakistan, there are diverse climatic zones and hence one devised solution might not be able to cope with different climates. Hence there is a need to explore the devised design in multiple climatic locations.

Managing the negative air pressure is important to ensure that there is outward outflow of air and it does not come back inside. This helps in infection control. Two air changes per hour are mostly desired but ensuring that there is no leakage of air movement is a key factor. There has to be a test of the facility through simulation at the design stage to ensure it functions properly as desired. Computational Fluid Dynamics (CFD) could be of great help. Prior to commissioning of the physical facility, testing of air tightness would be necessary to ensure its functional optimization for extensive usage.

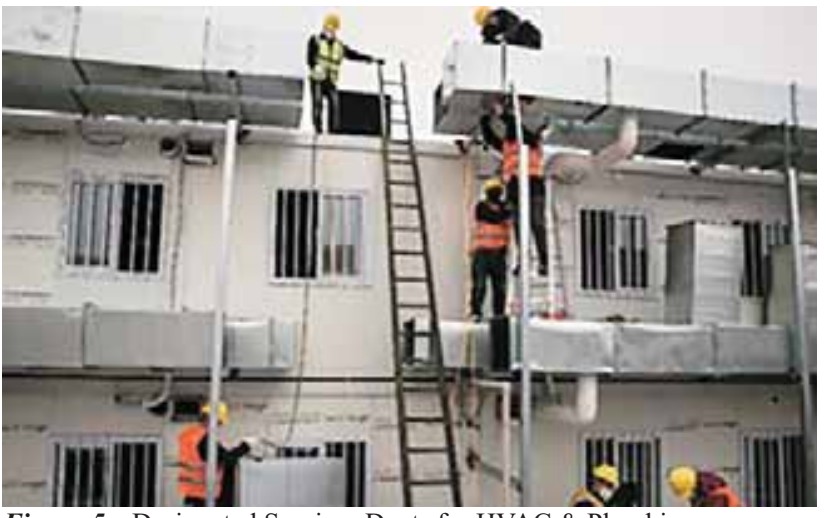

Figure-5: Designated Services Ducts for HVAC \& Plumbing. Source: Wuhan, 2020

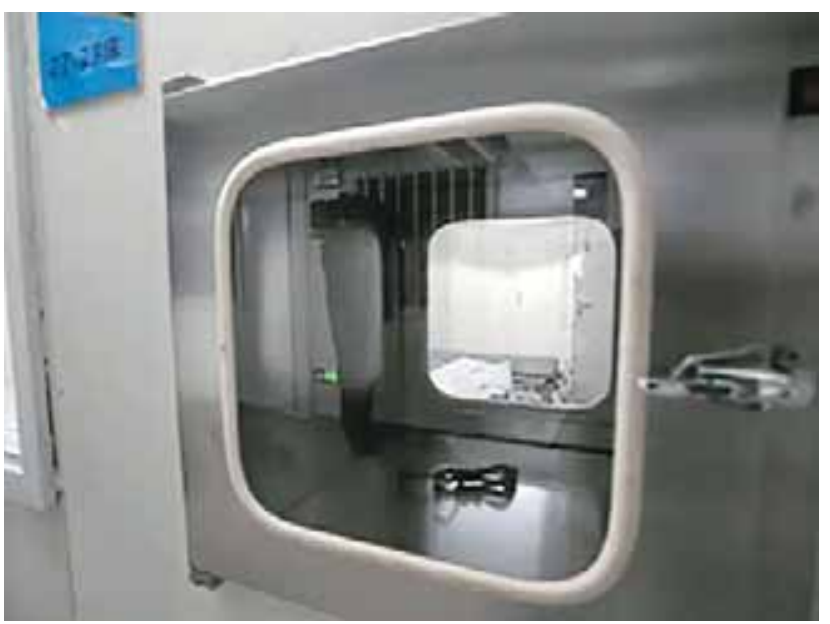

Figure-6: Double Sided opening Cabinets.

Source: Wuhan, 2020

Uni-directional air flow and intact movement can be a tedious job with regular mode of construction but with pre-fabricated strutures, its more easy to insulate or make them air tight. This important function, which lies at the heart of the design, requires forced ventilation systems. Since consistent and reliable energy supply, in the form of electricity, would act as the backbone of the functional stability, optimizing for renewable energy usage through solar energy, using uninterrupted power (UPS) supply system or even a backup generator could facilitate the need.

\section{PROPOSED DESIGN INTERVENTIONS}

Proposed design interventions using standardized shipping containers are shared below. This design incorporates the solutions for quarantine facilities and isolation wards/units with respect to the availability of spaces and land within preferred and designated hospitals for treating COVID-19 patients. There is need to incorporate the local climatic 
conditions methodologies and techniques that could be adopted to built them in as much time efficient manner as possible. This devised design should focus on ensuring the infection control processes and their implementation. Usage of Computational Fluid Dynamics (CFD) could be a very helpful tool for exploration of efficiency in these proposed design interventions.

\section{CONCLUSIONS:}

Fighting a pandemic is a great challenge for any advanced and developed nation across the globe. Such challenge for a developing country increases the magnitude multifolds.

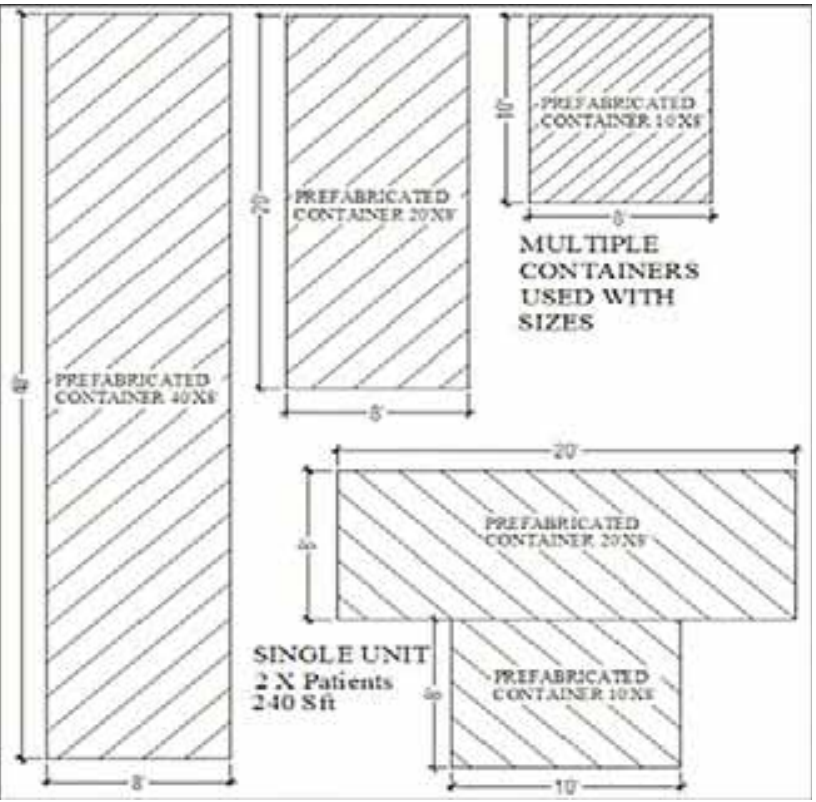

Figure-10: Multiple Shipping Containers used and Single Unit for Quarantine.
Hence addressing COVID-19 requires extreme measures. These will include quarantining the public where there is risk of the disease, with options of self and community isolation. Devising new strategies as per the need of the hour, engaging multiplayer approach where strategic and allied governmental bodies contribute to the cause, developing and enhancing the healthcare infrastructure along with improving the human capital, building confidence in the nation are all required. There has to be a well coordinated and planned line of action to be implemented and should be followed on to ensure healthcare infrastructure that may be able to cope with the challenge and allied departments/domains.

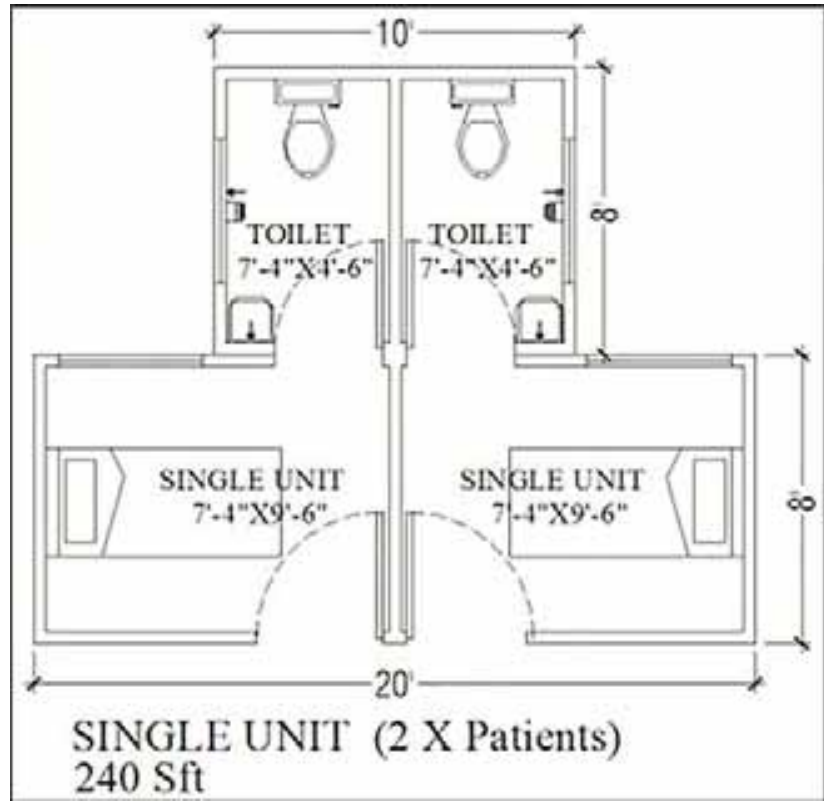

Figure-11: Architectural Plan for Single Unit Quarantine of Two Patients.

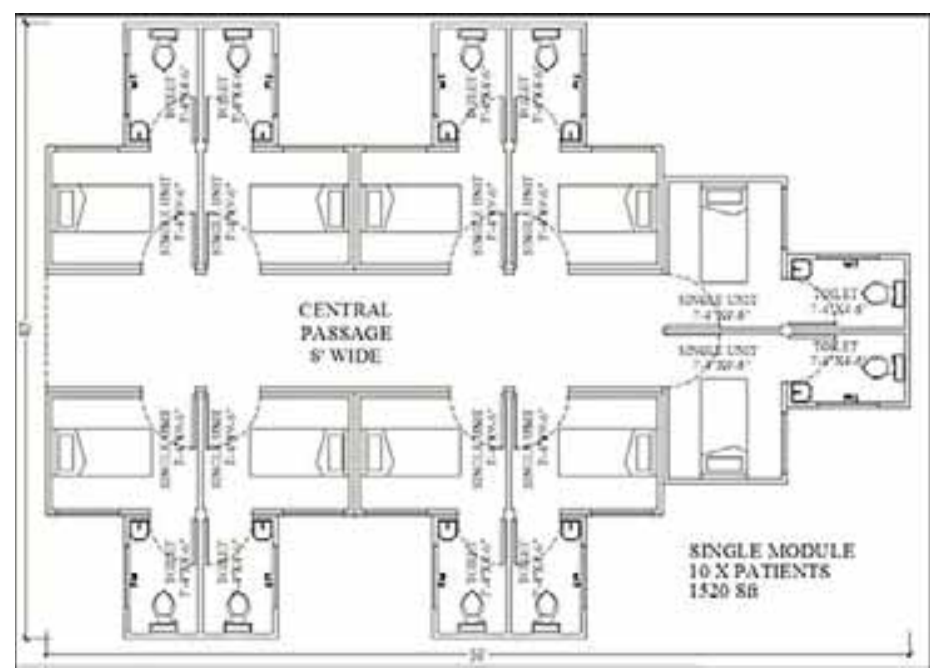

Figure-12: Architectural Plan for Single Module Quarantine of Ten Patients. 


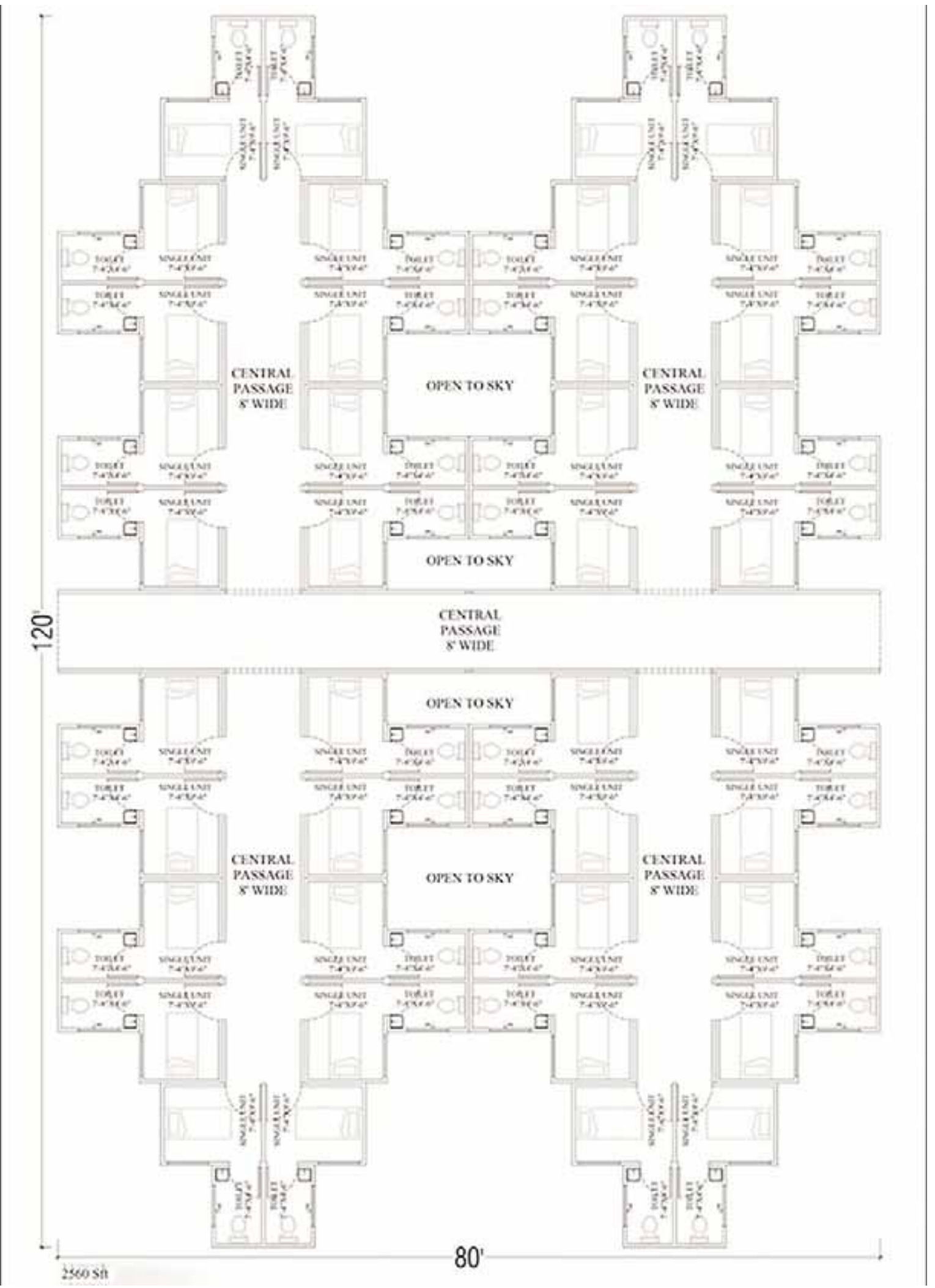

Figure-13: Architectural Plan for Four Module Quarantine of Forty Patients. 


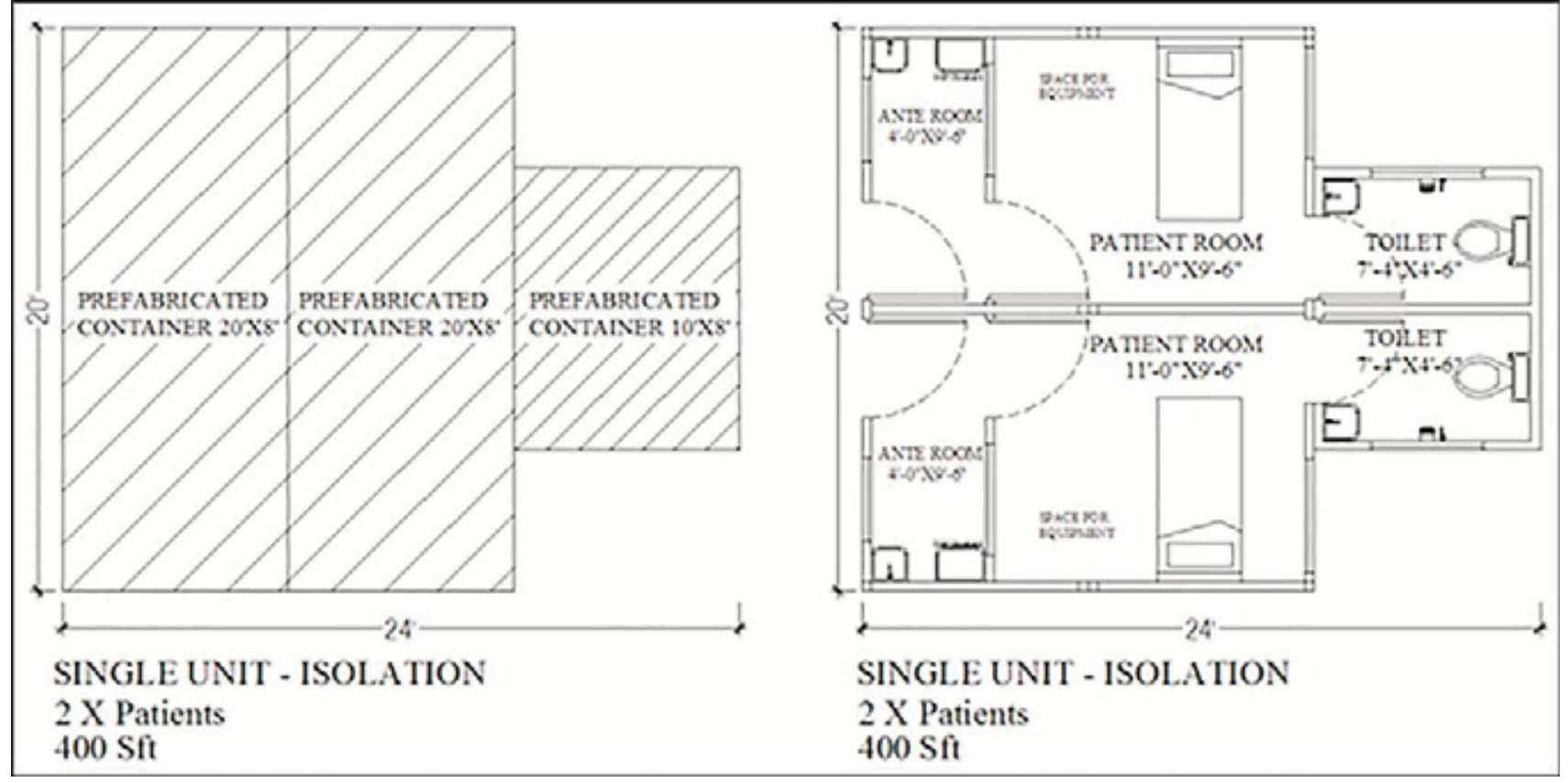

Figure-14: Multiple Shipping Containers used and Architectural Plan for Single Unit Isolation Room for Two Patients.

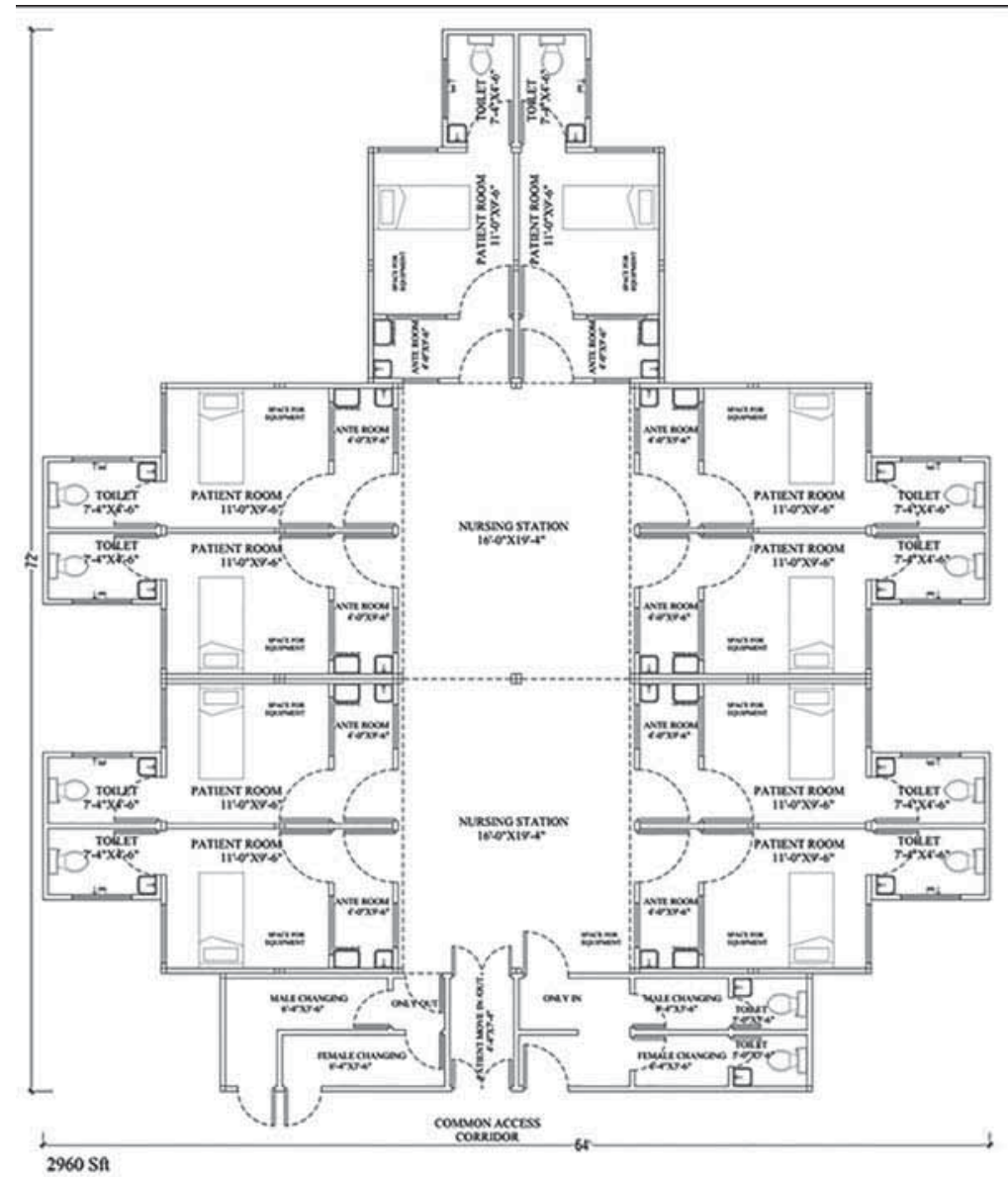

Figure-15: Architectural Plan for Single Module with Five Isolation Units of Ten Patients. 


\section{REFERENCES}

Ahmad, T., Khan, M., Khan, F. M., and Hui, J., 2020, “Are We Ready for the New Fatal Coronavirus: scenario of Pakistan?” Human Vaccines and Immunotherapeutics, $1-3$.

Bearman, G., Pryor, R., Albert, H., Brath, L., Britton, A., Cooper, K., ... Stevens, M. P., 2020, "Novel Coronavirus and Hospital Infection Prevention: Preparing for the Impromptu Speech", Infection Control and Hospital Epidemiology, 1-7.

Bedford, J., Farrar, J., Ihekweazu, C., Kang, G., Koopmans, M., \& Nkengasong, J., 2019, “A New Twenty-First Century Science for Effective Epidemic Response. Nature", 575(7781), 130-136.

Brüssow, H., 2020, “The Novel Coronavirus - A Snapshot of Current Knowledge. Microbial Biotechnology”, 0, 17517915.13557.

CDC, 2020a, "Interim Infection Prevention and Control Recommendations for Patients with Confirmed Coronavirus Disease 2019 (COVID-19) or Persons Under Investigation for COVID-19 in Healthcare Settings", Viewed from https://www.cdc.gov/coronavirus/2019-ncov/infection-control/controlrecommendations.html?CDC AA_refVal=https\%3 A\%2F\%2Fwww.cdc.gov\%2Fcoronavirus\%2F 2019 ncov\%2Fhcp\%2Finfection-control.html

CDC, 2020b, "Isolation and Quarantine Concepts", Viewed 23 Jan. 2020, from

Columbus, C., Brust, K. B., and Arroliga, A. C., 2020, "Novel Coronavirus: an Emerging Global Threat. Baylor University Medical Center Proceedings", 0(0), 1-4.

Conti, A. A., 2016, Quarantine through History. International Encyclopedia of Public Health

Ibrahim, I. M., Abdelmalek, D. H., Elshahat, M. E., \& Elfiky, A. A., 2020, "COVID-19 Spike-Host Cell Receptor GRP78 Binding Site Prediction", Journal of Infection.

Khan, S. A., 2019, "Situation Analysis of Health Care System of Pakistan: Post 18 Amendments. Health Care?", Current Reviews, 07(03), 1-9.

Khan, S., Siddique, R., Ali, A., Xue, M., and Nabi, G., 2020, "Novel Coronavirus, Poor Quarantine, and the Risk of Pandemic", Journal of Hospital Infection.

United Nations, 2020, "Considerations for Quarantine of Individuals in the Context of Containment for Coronavirus Disease", Viewed 12 Dec. 2019.

NIH, 2020a, "Designated Hospitals / Isolation Ward for COVID-19", Viewed 16 Feb. 2020 from . NIH, P., 2020b, “Advisory on Mitigation Strategies Covid-19”, Viewed 22 Jan. 2020 from

Stahl, M., and Falaschetti, D., 2012, "Public Sector. Encyclopedia of Health Care Management", WHO, 2020, "Novel Coronavirus Global Research and Innovation forum: Towards a Research Roadmap. Viewed 20 Jan. 2020 from https://www.who.int/docs/default-source/coronaviruse/global-research-forum-draft-agenda-feb-6.pdf

Wuhan, 2020,

Tsou, et. al., 2004, 